\title{
Automatic implantable cardioverter defibrillator: when not to implant
}

\author{
Maria Teresa La Rovere, Egidio Traversi \\ Department of Cardiology, Istituti Clinici Scientifici Maugeri IRCCS, Institute of Montescano (PV), Italy
}

\begin{abstract}
The implantable cardioverter-defibrillator (ICD) is the mainstay therapy for primary prevention of sudden cardiac death in patients with heart failure with a reduced ejection fraction. Current indications for prophylactic ICD are based on the results of randomized controlled trials dating back to 15-20 years ago, which have usually enrolled highly selected patients with few comorbidities and only a small number of patients aged $>75$ years. Existing literature suggest an age-dependent attenuation of the efficacy of the ICD. Because of the ageing of the population, there is need for data addressing device efficacy among older patients that also considers the impact of geriatric syndromes on health status. The assessment of frailty may be of value in identifying elderly patients who may or may not benefit from ICD placement for primary prevention of sudden cardiac death.
\end{abstract}

Correspondence: Maria Teresa La Rovere, Istituti Clinici Scientifici Maugeri IRCCS, Istituto di Montescano, 27040 Montescano (PV), Italy. E-mail: mariateresa.larovere@icsmaugeri.it

Key words: Implantable cardioverter defibrillator; older adults; primary prevention; frailty.

Contributions: All the authors made a substantive intellectual contribution. All the authors have read and approved the final version of the manuscript and agreed to be accountable for all aspects of the work.

Conflict of interest: The authors declare that they have no competing interests, and all authors confirm accuracy.

Availability of data and materials: The datasets used and/or analyzed during the current study are available from the corresponding author on reasonable request.

Received for publication: 3 January 2020.

Accepted for publication: 4 February 2020.

${ }^{\circ}$ Copyright: the Author(s), 2020

Licensee PAGEPress, Italy

Monaldi Archives for Chest Disease 2020; 90:1225

doi: 10.4081/monaldi.2020.1225

This article is distributed under the terms of the Creative Commons Attribution Noncommercial License (by-nc 4.0) which permits any noncommercial use, distribution, and reproduction in any medium, provided the original author(s) and source are credited.

\section{Introduction}

Effective risk stratification to identify patients at risk of developing life-threatening ventricular arrhythmia and sudden cardiac death (SCD) is likely the major unsolved area in clinical cardiology. Randomized trials demonstrating that mortality can be effectively reduced by prophylactic implantation of a cardioverter defibrillator (ICD) established the assessment of left ventricular ejection fraction as the gold standard risk predictor [1,2]. Current guidelines $[3,4]$ state that patients with left ventricular ejection fraction $\leq 30 \%$ or $\leq 35 \%$ with New York Heart Association Class II or III heart failure who are $>40$ days post myocardial infarction or $>90$ days post revascularisation and who have a reasonable expectation of survival for at least one year are eligible for a primary prevention ICD. However, left ventricular ejection fraction measurement has both limited sensitivity and specificity as a tool for arrhythmic risk stratification. Actually, the major burden of SCD occurs in patients with less severe degrees of left ventricular impairment and most important only one third of implanted patients had appropriate device therapy over the 3- to 5-year follow-up period. This raises concern that many patients are exposed to the risk of receiving inappropriate therapy or implant complications without any benefit. Thus, opinion leaders emphasise the need to remove the "stumbling block" of ejection fraction for arrhythmic risk stratification and to translate from the field of "high-risk ejection fraction" to the broader concept of the "high-risk patient" [5]. This, coupled with new technologies and improved knowledge, has promoted a renewed interest in a multiparametric approach to risk stratification that should consider the complexity of factors underlying sustained ventricular rhythms and sudden death [6]. This article will discuss some aspects of the current indications for which controversial evidence might call into question the role of primary prevention ICDs with a focus on elderly patients.

\section{Areas of concern with the current ICD indication}

One important area of concern is that the current indication for ICD implantation are based upon the findings of trials dating back to 15-20 years ago with medical treatment of patients not satisfying the current standard of optimized therapy. The rate of sudden death may be decreasing with improvements in guideline-directed medical therapy. This trend has been examined on data from 40,195 patients who had heart failure with reduced ejection fraction and were enrolled in any of 12 clinical trials spanning the period from 1995 through 2014 [7]. Sudden death was reported in 3583 patients. There was a $44 \%$ decline in the rate of sudden death across the trials $(\mathrm{p}=0.03)$. The cumulative incidence of sudden death at 90 days after randomization was $2.4 \%$ in the earliest trial and $1.0 \%$ in the most recent trial. 
Moreover, the pivotal trials upon which are based the current ICD indication have usually enrolled relatively young selected patients with few comorbidities. The real-world scenario is often in contrast with these characteristics as patients with left ventricular dysfunction are mainly in the age range 70 to 80 years, with frequent frailty and concomitant comorbidity. In a National Cardiovascular Data Registry ICD cohort [8], the median age of patients receiving ICDs was 68 years while the median age of the patients enrolled in the MADIT II and SCD-HeFT trial [1,2] were 64 years and 60 years of age, respectively. Patients in the ICD cohort [8] also had worse renal function, more diabetes and other comorbidities.

Among comorbidities, evolving evidence suggest that the potential risks and benefits of ICDs should be carefully considered in patients with chronic renal failure [9]. In a secondary analysis of the MADIT-II study, patients with creatinine $\geq 2.5 \mathrm{mg} / \mathrm{dL}$ and/or blood urea nitrogen $(\mathrm{BUN}) \geq 50 \mathrm{mg} / \mathrm{dL}$ did not benefit from the ICD because of very high overall mortality [10]. In a large, contemporary, noninterventional study of community-based patients with heart failure and chronic kidney disease, ICD placement was not significantly associated with improved survival but was associated with increased risk for subsequent hospitalization due to heart failure (adjusted relative risk: 1.49; 95\% CI: 1.33-1.60) and all-cause hospitalization (adjusted relative risk, 1.25; 95\% CI, 1.201.30) [11].

Similarly, in patients with atrial fibrillation the mortality benefit of ICD remains unclear. In a recent systematic review and metaanalysis including 25 observational studies with over 60,000 patients [12], overall mortality and appropriate shock therapy were higher in ICD patients with atrial fibrillation as compared to normal sinus rhythm. Moreover, there was no difference in mortality between atrial fibrillation patients with ICD and patients who were only on goal directed medical therapy.

Comorbidity burden also impact on the risk of inappropriate therapy. In a retrospective cohort study including 2235 patients (mean age $=69 \pm 11$ years, $75 \%$ men) from seven US healthcare delivery systems the median number of comorbidities was 6 (interquartile range $=4-8$ ), with $98 \%$ of patients having at least two comorbidities [13]. During a mean 2.2 years of follow-up, almost 10 $\%$ of patients experienced at least one inappropriate therapy. Higher comorbidity burden was associated with an increased risk of first inappropriate therapy with and adjusted hazard ratio [HR] of 1.94 [95\% CI: 1.14-3.31] for 4-5 comorbidities; of 2.25 [95\% CI: 1.25 4.05] for 6-7 comorbidities and of 2.91 [95\% CI: $1.54-5.50$ ] for 8-16 comorbidities. Patients with 8-16 comorbidities also had a higher risk of receiving inappropriate $v s$ appropriate therapy (Relative Risk, RR $1.74,95 \%$ CI: $1.07-2.82$ ).

Indeed, while stressing that all proposed treatment schemes should be adapted in view of comorbidities, life expectancy and the impact on quality of life, the guidelines remain however generic. The sentence "in the presence of a life expectancy of at least 1 year in good functional condition" lacks of definition or reference value of a "good functional condition". Several tools can help identifying clinically important variations in the magnitude of the survival benefit expected for patients with a class I indication for a primary prevention ICD. Particularly relevant are the results of two post-hoc analyses of the same randomized studies on which the recommendations are based upon $[10,14]$. In the MADIT-II posthoc study, Goldenberg et al. [10] identified a U-shaped relationship among five clinical variables (NYHA class $>2$, atrial fibrillation, QRS duration $>120 \mathrm{~ms}$, age $>70$ years and BUN $>26$ or $<50 \mathrm{mg} / \mathrm{dL}$ ) and the survival benefit from the ICD. While the ICD significantly improved the survival of patients with 1 or 2 "risk factors", no survival benefit was obtained from patients with either none or $\geq 3$ risk factors because their mortality was either too low or too high.

In the SCD-HeFT post-hoc analysis, Levy et al. [14] examined the relationship between baseline predicted mortality risk and the relative and absolute survival benefit of ICD treatment by applying a modification of the Seattle Heart Failure Model (SHFM). Dividing the study population in quintiles of risk, ICD treatment reduced mortality by approximately $50 \%$ in the lowest-risk group (predicted annual mortality $\leq 5 \%$ ), whereas there was no ICD benefit in the highest-risk group where the predicted annual mortality exceeded $25 \%$. The combined use of the SHFM and the Seattle Proportional Risk Model (specifically developed to examine the proportion of mortality risk attributable to sudden death) identified patients less likely to derive a survival benefit from primary prevention ICDs in a large cohort of patients with and without ICDs [15].

The issue whether primary prevention ICD has a survival benefit in female, is a complex one. There are known sex differences in cardiac electrophysiology leading to a lower rate of sudden cardiac death in women [16]. Current ICD guidelines make no distinction between recommendations for ICD by gender although women were severely under-represented in the trials leading to the guideline indications. Indeed, women were $16 \%$ in the MADIT-II trial [1] and 23\% in the SCD-HeFT trial [2]. In the SCD-HeFT trial, a survival benefit of ICD therapy was not observed in women. The more advanced age of women as well as their increased comorbidity burden may suggest an increased competing risk of non-arrhythmic death as a possible explanation. A recent analysis on registry data from 11 European countries including more than 5033 ICD implantations between 2012 and 2014 does not support the former hypothesis [17]. Women did not differ in age or other clinical characteristics from men, but had a significantly lower mortality rate and received fewer appropriate ICD shocks [17].

Finally, the recent publication of the DANISH trial casts doubt on the role of primary prevention ICDs in patients with nonischemic cardiomyopathy [18]. The DANISH trial showed a reduction in SCD, but not in all-cause mortality in patients randomly assigned to an ICD. However, a secondary analysis showed a significant association between reduced all-cause mortality and ICD in patients $\leq 70$ years of age (HR, $0.70 ; 95 \% \mathrm{CI}, 0.51-0.96 ; \mathrm{p}=0.03$ ) but not in patients $>70$ years of age (HR, 1.05 ; 95\% CI, 0.68 $1.62 ; \mathrm{p}=0.84$ ) [19].

\section{Primary prevention ICDs in the elderly}

The question of whether the ICD is beneficial in elderly patients is of great importance. Although current guidelines do not propose any age limit for the primary prevention ICDs, some have questioned the role of the ICD in the elderly arguing that these patients were not well represented in the pivotal trials. Indeed, the median age of the patients enrolled in the MADIT II and SCD-HeFT trial $[1,2]$ were 64 years and 60 years of age, respectively, and patients older than 75 years accounted for only $10 \%$ of the studied populations.

Evidence for the benefit of ICD in preventing sudden cardiac death in older adults can be derived from meta-analysis of the randomized data and observational studies. In one meta-analysis [20], where the age cut-off was set at 60 years, it was found that prophylactic ICD therapy significantly reduced mortality in elderly patients (HR, 0.75 [95\% CI, 0.61 to 0.91]) although the survival benefit was smaller than in younger patients (HR, 0.65 [95\% CI, 0.50 to 0.83$]$ ). The age-dependent attenuation of the efficacy of the ICD was confirmed by a second meta-analysis merging data from 
5 major ICD trials [21]. However, is worth to emphasize that sample sizes were limited among patients aged $\geq 75$ years. The impact of ICD implantation for primary prevention in older patients was recently addressed in a meta-analysis of predominantly observational data [22] by using several age ranges: 70 to 84 years, 75 to 84 years, 79 to 90 years, and $\geq 75$ years. Again, a survival advantage of ICD versus no ICD was seen with an overall HR of 0.75 (95\% CI: 0.67 to $0.83 ; p<0.001)$. A recent multicentre French study assessing outcomes among 150 patients aged $\geq 80$ years of whom $76 \%$ had no more than one associated comorbidity supports the concept that the ICD is of potential relevance to prevent sudden cardiac death in well selected older individual [23].

The already quoted secondary analysis from the DANISH study [19] further underscores the importance of comorbidities in attenuating the survival benefit of primary prevention ICDs in the elderly. The patients $>70$ years old enrolled in the study had a significantly higher prevalence of atrial fibrillation, hypertension and lower glomerular filtration rate. They also had higher median Nterminal pro-brain natriuretic peptide and longer median QRS duration. Among these patients, the risk of SCD and non-SCD was $1.6(0.8-3.2)$ and $5.4(3.7-7.8)$ events/100 patient-years, respectively whereas in patients $\leq 70$ years the risk of SCD and non-SCD was 1.8 (1.3-2.5) and 2.7 (2.1-3.5) events/100 patient-years, respectively.

Although the 2017 American Heart Association/American College of Cardiology/Heart Rhythm Society guideline [4] highlight the need for a careful evaluation of comorbidities in the decisionmaking for ICD implantation in elderly patients, the assessment of "a life expectancy greater than 1 year" is even more challenging with advancing age for the occurrence of geriatric syndromes. In other words, although the comorbidity burden generally increases with advancing age, the two are not linearly related and each of them has an independent impact on the survival benefit of the ICD. Moreover, chronologic age, by itself, is seldom a reliable estimate of an individual's health status. This is particularly relevant for elderly patients where the presence of geriatric syndromes increases the variation in health status. The assessment of frailty may help better defining life expectancy in elderly patients [24].

Frailty, defined as a clinically recognizable state of increased vulnerability resulting from aging-associated decline in reserve and function across multiple physiologic systems, is increasingly recognised as an important predictor in heart failure [25]. A recent systematic review explored whether frailty may help identify patients in whom an ICD does not improve overall mortality risk [26]. Frailty was defined broadly using any validated single component (e.g., walking speed, weight loss) or multi-component tool (e.g., cumulative deficit index). Nine articles met the inclusion criteria. All studies indicated that mortality was higher amongst individuals identified as frail, regardless of definition. In a prespecified subgroup of SCA-HeFT trial [27] including 2397 patients who performed a six-minute walking test before randomization, those patients in the lower tertile of walking distance did not benefit from ICD therapy after 3 years. A cohort of 83,792 Medicare beneficiaries in an ICD registry reported higher 1-year mortality following ICD in those with frailty or dementia [28].

\section{Conclusions}

The use of ICDs in elderly patients should be individualised and carefully scrutinised. It should take into consideration overall health status, symptom severity, co-morbidities and intermediate and longterm prognosis. Existing literature suggests that individuals with frailty may not benefit from ICD placement for primary prevention of SCD. Frailty evaluation may likely represent a useful refinement of life expectancy definition. Further studies are however needed to incorporate frailty evaluation in the routine assessment of candidates to ICD implantation.

\section{References}

1. Moss AJ, Zareba W, Hall WJ, et al. Prophylactic implantation of a defibrillator in patients with myocardial infarction and reduced ejection fraction. N Engl J Med 2002;346:877-83.

2. Bardy GH, Lee KL, Mark DB, et al. Amiodarone or an implantable cardioverter-defibrillator for congestive heart failure. N Engl J Med 2005;352:225-37.

3. Priori SG, Blomström-Lundqvist C, Mazzanti A, et al. 2015 ESC Guidelines for the management of patients with ventricular arrhythmias and the prevention of sudden cardiac death: The Task Force for the Management of Patients with Ventricular Arrhythmias and the Prevention of Sudden Cardiac Death of the European Society of Cardiology (ESC). Eur Heart J 2015;36:2793-867.

4. Al-Khatib SM, Stevenson WG, Ackerman MJ, et al. 2017 AHA/ACC/HRS Guideline for Management of Patients With Ventricular Arrhythmias and the Prevention of Sudden Cardiac Death: Executive Summary. Circulation 2018;138:e210-71.

5. Chugh SS. Sudden cardiac death in 2017: Spotlight on prediction and prevention. Int J Cardiol 2017;237:2-5.

6. Disertori M, Gulizia MM, Casolo G, et al. Improving the appropriateness of sudden arrhythmic death primary prevention by implantable cardioverter-defibrillator therapy in patients with low left ventricular ejection fraction. Point of view. J Cardiovasc Med (Hagerstown) 2016;17:245-55.

7. Shen L, Jhund PS, Petrie MC, et al. Declining risk of sudden death in heart failure. N Engl J Med 2017;377(41-51.

8. Kramer DB, Kennedy KF, Noseworthy PA, et al. Characteristics and outcomes of patients receiving new and replacement implantable cardioverter-defibrillators: results from the NCDR. Circ Cardiovasc Qual Outcomes 2013;6:488-97.

9. Pun PH, Al-Khatib SM, Han JY, et al. Implantable cardioverterdefibrillators for primary prevention of sudden cardiac death in CKD: a meta-analysis of patient-level data from 3 randomized trials. Am J Kidney Dis 2014;64:32-9.

10. Goldenberg I, Vyas AK, Hall WJ, et al. Risk stratification for primary implantation of a cardioverter-defibrillator in patients with ischemic left ventricular dysfunction. J Am Coll Cardiol 2008;51:288-96.

11. Bansal N, Szpiro A, Reynolds K, et al. Long-term outcomes associated with implantable cardioverter defibrillator in adults with chronic kidney disease. JAMA Intern Med 2018;178:390-8.

12. Mustafa U, Dherange P, Reddy R, et al. Atrial fibrillation is associated with higher overall mortality in patients with implantable cardioverter-defibrillator: A systematic review and meta-analysis. J Am Heart Assoc 2018;7:e010156.

13. Hajduk AM, Gurwitz JH, Tabada G, et al. Influence of multimorbidity on burden and appropriateness of implantable cardioverter-defibrillator therapies. J Am Geriatr Soc 2019;67:1370-8.

14. Levy WC, Lee KL, Hellkamp AS, et al. Maximizing survival benefit with primary prevention implantable cardioverterdefibrillator therapy in a heart failure population. Circulation 2009; 120:835-42. 
15. Bilchick KC, Wang Y, Cheng A, et al. Seattle Heart Failure and Proportional Risk Models predict benefit from implantable cardioverter-defibrillators. J Am Coll Cardiol 2017;69:2606-18.

16. Gillis AM. Atrial fibrillation and ventricular arrhythmias: Sex differences in electrophysiology, epidemiology, clinical presentation, and clinical outcomes. Circulation 2017;135:593608.

17. Sticherling C, Arendacka B, Svendsen JH, et al. Sex differences in outcomes of primary prevention implantable cardioverterdefibrillator therapy: combined registry data from eleven European countries. Europace 2018;20:963-70.

18. Køber L, Thune JJ, Nielsen JC, et al. Defibrillator implantation in patients with nonischemic systolic heart failure. N Engl J Med 2016;375:1221-30.

19. Elming MB, Nielsen JC, Haarbo J, et al. Age and outcomes of primary prevention implantable cardioverter-defibrillators in patients with nonischemic systolic heart failure. Circulation 2017; 136:1772-80.

20. Santangeli P, Di Biase L, Dello Russo A, et al. Meta-analysis: age and effectiveness of prophylactic implantable cardioverterdefibrillators. Ann Intern Med 2010;153:592-9.

21. Hess PL, Al-Khatib SM, Han JY, et al. Survival benefit of the primary prevention implantable cardioverter-defibrillator among older patients: does age matter? An analysis of pooled data from 5 clinical trials. Circ Cardiovasc Qual Outcomes 2015;8:179-86.
22. Kusumoto FM, Bailey KR, Chaouki AS, et al. systematic review for the 2017 AHA/ACC/HRS Guideline for management of patients with ventricular arrhythmias and the prevention of sudden cardiac death. Circulation 2018;138:e392-414.

23. Zakine C, Garcia R, Narayanan K, et al. Prophylactic implantable cardioverter-defibrillator in the very elderly. Europace.2019;21:1063-19.

24. Strandberg TE, Nieminen T. Future perspectives on the role of frailty in cardiovascular diseases. Adv Exp Med Biol 2020;1216:149-52.

25. Vigorito C, Abreu A, Ambrosetti M, et al. Frailty and cardiac rehabilitation: A call to action from the EAPC Cardiac Rehabilitation Section. Eur J Prev Cardiol 2017;24:577-90.

26. Chen MY, Orkaby AR, Rosenberg MA, et al. Frailty, implantable cardioverter defibrillators, and mortality: a systematic review. J Gen Intern Med 2019;34:2224-31.

27. Fishbein DP, Hellkamp AS, Mark DB, et al. Use of the 6-min walk distance to identify variations in treatment benefits from implantable cardioverter-defibrillator and amiodarone: results from the SCD-HeFT (Sudden Cardiac Death in Heart Failure Trial). J Am Coll Cardiol 2014;63:2560-8.

28. Green AR, Leff B, Wang Y, et al. Geriatric conditions in patients undergoing defibrillator implantation for prevention of sudden cardiac death: Prevalence and impact on mortality. Circ Cardiovasc Qual Outcomes 2016;9:23-30. 